LiVINGSTONE, D., and A. WAGNER

Math. Zeitschr. 90, 393-403 (1965)

\title{
Transitivity of finite permutation groups on unordered sets
}

By

DONALd Livingstone and Ascher WAGNer

\section{§1. Introduction}

From any given permutation group acting on a finite collection of $n$ points one can form, for each positive integer $k \leqq n$, two permutation groups by considering respectively the permutations induced by the given group on the unordered sets of $k$ distinct points and those induced on the ordered sets of $k$ distinct points. We consider relations between these two groups. Our main object is to prove Theorem 2 below.

In $\S 1$ we establish the following theorem.

Theorem 1. If $k \geqq 2$ and $2 k \leqq n$, then the number of orbits of the group on the unordered sets of $k$ points is at least as great as the number of orbits on the unordered sets of $k-1$ points.

Using this result we deduce the following relation between the two groups.

Theorem 2. If $k \geqq 2,2 k \leqq n$ and the group on the unordered sets of $k$ points is transitive then

(a) the group on the ordered sets of $k-1$ distinct points is transitive (i.e. the given permutation group is $(k-1)$-transitive), and

(b) if also $k \geqq 5$, the group on the ordered sets of $k$ points is transitive (i.e. the given permutation group is $k$-transitive).

Transitivity on unordered sets has been considered from time to time by various writers, e. g. MILLER [12]. HUGHES [9] has recently proved Theorem 2(a) under the stronger hypothesis $k ! \leqq n .{ }^{1}$ )

It has been shown by Beaumont and PeTErson [1] that if, for all $k \leqq n$, the group on the unordered sets of $k$ points is transitive then the group is, in general, the full symmetric group or the alternating group. Exceptions occur only with the following groups:

(i) $n=5$ : the affine group $A_{1}(5)$;

(ii) $n=6: P G L_{2}(5)$;

(iii) $n=9: P G L_{2}(8)$;

(iv) $n=9: P \Gamma L_{2}(8)$.

This result may be deduced from our treatment, as is shown in $\S 4$.

1) The question has also been considered by WIELANDT, who showed in 1959, and privately communicated to the authors, that if $k \geqq 3$ and $2 k \leqq n$, then $G$ is at least doubly transitive. 
It is clear that the condition $2 k \leqq n$ is necessary for any statement having the form of Theorem 2 . We show in $\S 6$ that the additional condition $k \geqq 5$ in Theorem 2(b) cannot be omitted.

\section{§2. General definitions and notations}

We develop the notation introduced by WIELANDT $[13,14]$. We consider a finite set $\Omega$ consisting of $n$ elements called points. We shall use Greek letters $\alpha, \beta, \ldots$ to denote points; $S^{\Omega}$ and $A^{\Omega}$ denote respectively the symmetric and the alternating group on $\Omega$.

If $G$ is a group of permutations of the points of $\Omega$ and $1 \leqq k \leqq n$, we say that $G$ is $k$-transitive if $G$ induces a transitive group of permutations on the ordered sets of $k$ distinct points, and that $G$ is $k^{*}$-transitive if it induces a transitive group on the unordered sets of $k$ points.

If $\Delta$ is a subset of $\Omega$ we denote by $G_{[d]}$ the subgroup of $G$ fixing $\Delta$ pointwise. We shall also call $G_{[\Delta]}$ the stabilizer of $\Delta$. If the stabilizer of every subset of $k$ points of $\Omega$ is the identity we shall say that $G$ is $k$-regular on $\Omega$. If $G$ is $k$-transitive and $k$-regular on $\Omega$ we shall say that $G$ is sharply $k$-transitive on $\Omega$. The subgroup of $G$ fixing $\Delta$ as a set will be denoted by $G_{(\Delta)}$. If the elements of $\Omega$ have a natural order we use $G_{[k]}$ and $G_{(k)}$ for the corresponding groups formed with respect to the first $k$ points of $\Omega$.

If $G$ is an intransitive group on $\Omega$ and $\Gamma$ is a union of orbits of $G$ then $G^{\Gamma}$ denotes the homomorphic image of $G$ obtained as a permutation group by restricting $G$ to $\Gamma$.

As is customary we shall denote the symmetric and alternating group on $k$ points also by $S_{k}$ and $A_{k}$ respectively. The Mathieu groups on 11 and 12 points we shall denote by $M_{11}$ and $M_{12}$ respectively. We shall denote the group of all linear transformations of the projective line over the field with $q$ elements by $P G L_{2}(q)$. The corresponding group of all semilinear transformations we shall denote by $P \Gamma L_{2}(q)$. The one-dimensional linear and semilinear affine groups, regarded as permutation groups on the $q$ finite points of the line over the field of $q$ elements, will be denoted by $A_{1}(q)$ and $\Gamma A_{1}(q)$ respectively.

\section{§ 3. Proof of Theorem 1}

The proof of Theorem 1 will be based upon the description of the irreducible characters of $S^{\Omega}$ given by FroBENIUS [5]. The character formulae enable us to compare the number of orbits on unordered sets of $k$ points with the number on sets of $k^{\prime}$ points. It is first necessary to translate the enumeration problem to character-theoretic terms. This is effected by Lemmas 1 and 2 below.

Lemma 1. Let $\Delta$ and $\Gamma$ form a partition of $\Omega: \Delta \cup \Gamma=\Omega, \Delta \cap \Gamma=\emptyset$, and let the number of points in $\Delta$ be $k$. Then the permutation group derived from $G$ by its action on the unordered sets of $k$ points is permutation isomorphic to the restriction to $G$ of the permutation representation of $S^{\Omega}$ on the cosets of $S_{(\Delta)}^{\Omega}$. 
To see the truth of this statement it suffices to consider the mapping $f: a \rightarrow a(\Delta)$ defined on $S^{\Omega} . f(a)=f(b)$ if and only if $a^{-1} b \in S_{(\Delta)}^{\Omega}$. Since $S^{\Omega}$ is $k$-transitive all unordered sets appear in the image. Furthermore $f(a b)=a f(b)$. The group on the unordered sets is by definition the restriction of the image of $S^{\Omega}$.

Lemma 2. If $K$ and $L$ are subgroups of a group $H$ and if, for each irreducible representation $Z_{\lambda}$ of $H, c_{\lambda}$ and $d_{\lambda}$ denote the multiplicities of the identity representations of $K$ and $L$ in the restrictions to these respective groups of the representation $Z_{\lambda}$ of $H$, then the number of orbits of the restriction to $K$ of the permutation representation of $H$ on the cosets of $L$ is given by the sum $\sum c_{\lambda} d_{\lambda}$.

In fact the permutation representation of $H$ on the cosets of $L$ is the linear representation induced on $H$ by the identity representation of $L$ and therefore, by the Frobenius reciprocity law, decomposes as $\sum d_{\lambda} Z_{\lambda}$. The restriction of the permutation representation to $K$ contains the identity representation exactly once for each orbit of $K$. Hence the total number of orbits of $K$ is equal to the multiplicity of the identity representation in the restriction to $K$, which is $\sum c_{\lambda} d_{\lambda}$.

Since the numbers $c_{\lambda}$ of Lemma 2 are non-negative integers, Theorem 1 is an immediate consequence of the following lemma.

Lemma 3. If, in the notation of Lemmas 1 and 2, we take $H=S^{\Omega}$ and $L=S_{(4)}^{\Omega}$ then the numbers $d_{\lambda}$ are all 0 or 1 . Moreover if $\Delta \supset \Delta^{\prime}$ and $2 k \leqq n$, and if $d_{\lambda}^{\prime}$ is the number corresponding to $d_{\lambda}$ when $\Delta$ is replaced by $\Delta^{\prime}$, then $d_{\lambda}^{\prime} \leqq d_{\lambda}$.

General decomposition theorems have been given for the Kronecker products of representations of $S^{\Omega}$ and the above lemma can be deduced from these. We shall instead outline a direct analytical proof based on the description of the irreducible representations in FroBENIUS [5].

Let $\lambda$ denote the partition $\left[\lambda_{1}, \lambda_{2}, \ldots, \lambda_{n}\right]$ of $n$ with parts written in descending order and with $\lambda_{i} \geqq 0$, and let $s_{i}$ denote the $i^{\text {th }}$ partial sum of the sequence of parts. For each $r, 1 \leqq r \leqq n$, let $h_{r}$ be the sum of all the monomial functions of degree $r$ in a set of indeterminates $x_{1}, \ldots, x_{n}$; define $h_{0}=1$. Finally let $\delta_{\lambda}$ and $\delta_{0}$ denote the $n \times n$ determinants

$$
\delta_{\lambda}=\left|x_{j}^{i+\lambda_{n-i+1}}\right|, \quad \delta_{0}=\left|x_{j}^{i}\right| .
$$

If $\Lambda$ is the set of all partitions of $n$, we define three sets indexed by $\Lambda$ as follows:

$$
S_{\lambda}=\cap S_{\left(s_{i}\right)}^{a} ; \quad h_{[\lambda]}=\prod_{i=1}^{n} h_{\lambda_{i}} ; \quad h_{\{\lambda\}}=\frac{\delta_{\lambda}}{\delta_{0}},
$$

where the index $i$ runs from 1 to $n$. Then $\left\{h_{[\lambda]}: \lambda \in \Lambda\right\}$ and $\left\{h_{\{\lambda\}}: \lambda \in \Lambda\right\}$ form two bases for the vector space of rational integral symmetric functions of degree $n$, and FrobenIUS proved in [5], p. 517-520, that the irreducible representations $Z$ of $S^{\Omega}$ can be indexed by $\Lambda$ so that the identity representation of $S_{\mu}, \mu \in A$, induces $\sum d_{\lambda} Z_{\lambda}$ where $d_{\lambda}$ is the $h_{\{\lambda\}}$-coordinate of $h_{[\mu]}$. For the 
lemma we require the coefficients $d_{\lambda}$ when $\mu=[n-k, k]$ and $2 k \leqq n$. But by a theorem of JACOBI [8] we have, if $2 r \leqq n$,

$$
h_{\{n-r, r\}}=\left|\begin{array}{cc}
h_{r} & h_{r-1} \\
h_{n-r+1} & h_{n-r}
\end{array}\right| .
$$

By summation from $r=1$ to $k$ we obtain because of $h_{0}=1$ and $h_{n}=h_{\{n\}}$ :

$$
\sum_{r=0}^{k} h_{\{n-r, r\}}=h_{[n-k, k]},
$$

from which the lemma follows.

\section{§ 4. Proof of Theorem 2 (a)}

If a group $G$ is $k^{*}$-transitive on a set $\Omega$ then the stabilizers of the subsets of $k$ points are conjugate. Our next four lemmas connect properties of stabilizers with multiple transitivity. The first of these lemmas is well known.

Except in Lemma 8 we do not assume that $2 k \leqq n$.

Lemma 4. Let $G$ be a permutation group on a set $\Omega$ of $n$ points. Let $1 \leqq k<n$. If the stabilizer of every subset of $k-1$ points is transitive on the remaining $n-k+1$ points, then $G$ is $k$-transitive on $\Omega$.

The proof is obvious.

Lemma 5. Let $G$ be a permutation group on a set $\Omega$ and let $\gamma$ and $\delta$ be two points of $\Omega$ and $p$ a given prime. If there exist subgroups $C$ and $D$ of $G$ each of order a power of $p$ and having as their only fixed point $\gamma$ and $\delta$ respectively, then $\gamma$ and $\delta$ belong to the same orbit of $G$.

Proof. Let us suppose that $\gamma$ and $\delta$ belong to different orbits. Denote these by $\Gamma$ and $\Delta$ respectively. By considering the orbits of $C$ it follows immediately that $|\Gamma| \equiv 1(p)$ and $|\Delta| \equiv 0(p)$. Also by considering the orbits of $D$ it follows that $|\Gamma|=0(p)$ and $|\Delta| \equiv 1(p)$. This contradiction shows that $\gamma$ and $\delta$ belong to the same orbit.

Lemma 6. Let $G$ be a permutation group on a set $\Omega$ of $n$ points; let $1 \leqq k<n$, and let $p$ be a given prime. If for every subset $\Gamma$ of $\Omega$ with $|\Gamma|=k$, there exists a subgroup $C$ of $G$ which has order a power of $p$ and which fixes the points of $\Gamma$ and no further points, then $G$ is $k$-transitive on $\Omega$.

Proof. Let $\Sigma$ be any subset of $\Omega$ containing $k-1$ points and let $\gamma$ and $\delta$ be any two points of $\Omega-\Sigma$. Further let $C$ and $D$ be subgroups of $G_{[\Sigma]}$, each of order a power of $p$, fixing $\gamma$ and $\delta$ respectively and no further points of $\Omega-\Sigma$. Then by considering $C$ and $D$ as permutation groups on $\Omega-\Sigma$ it follows from Lemma 5 that $\gamma$ and $\delta$ belong to the same orbit of $G_{[\Sigma]}$. Hence $G_{[\Sigma]}$ is transitive on $\Omega-\Sigma$ and it follows from Lemma 4 that $G$ is $k$-transitive on $\Omega$. 
Lemma 7. Let $G$ be a permutation group on a set $\Omega$ and let $s$ and $t$ be given integers with $s>1$. If the stabilizer of every subset of $k-1$ points has order $s$ and the stabilizer of every subset of $k$ points has order $t$, then $G$ is $(k-1)$ transitive on $\Omega$.

Proof. Let $\Sigma$ be any subset of $\Omega$ containing $k-1$ points. Then clearly $G_{[\Sigma]}$ splits $\Omega-\Sigma$ into orbits of length $s / t$. Moreover $s / t>1$ since otherwise $G_{[\Sigma]}$ is the identity and $s=1$. Now let $p$ be a prime dividing $s / t$. Then, if $P$ is a Sylow $p$-subgroup of $G_{[\Sigma]}$, the subgroup $P$ fixes the points of $\Sigma$ only. The result now follows immediately from Lemma 6.

We are now able to prove the "general" case of Theorem 2(a).

Lemma 8. Let $G$ be a $k^{*}$-transitive permutation group on a set $\Omega$ of $n$ points, with $2 k \leqq n$. Then, if the stabilizer of a subset of $k-1$ points is not the identity, $G$ is $(k-1)$-transitive on $\Omega$.

Proof. It follows immediately from Theorem 1 and the definition of $k^{*}$-transitivity that $G$ is $(k-1) *$-transitive on $\Omega$. Consequently all the stabilizers of the subsets of $k$ points have the same order and also the stabilizers of the subsets of $k-1$ points have the same order which, by our assumption, is greater than 1 . We may now apply Lemma 7.

Our next lemma enables us to eliminate those cases of Theorem 2(a) not covered by Lemma 8 .

Lemma 9. Let $k \geqq 3$. Let $G$ be a permutation group on a set $\Omega$ of $n$ points which is $k^{*},(k-1)^{*}$ and $(k-2)$ but not $(k-1)$-transitive on $\Omega$. Then $n=k$ and $G=A^{\Omega}$ except in the following cases:

$$
\begin{array}{lll}
n=5, & k=4, & G=A_{1}(5), \\
n=6, & k=5, & G=P G L_{2}(5), \\
n=9, & k=5, & G=P G L_{2}(8), \\
n=9, & k=5, & G=P \Gamma L_{2}(8) .
\end{array}
$$

Proof. Let $\Sigma$ be a subset of $k-1$ points and $\Gamma$ a subset of $k$ points. It follows from Lemma 7 that $G_{[\Sigma]}$, hence also $G_{[\Gamma]}$, is the identity. Consequently $G_{(\Sigma)} \cong G_{(\Sigma)}^{\Sigma}$ and $G_{(\Gamma)} \cong G_{(\Gamma)}^{\Gamma}$.

By considering $G$ as a permutation group on the unordered sets of $k$ points we obtain

$$
|G|=\left(\begin{array}{l}
n \\
k
\end{array}\right) a \text { where } \quad a=\left|G_{(I)}^{\Gamma}\right| .
$$

Also by considering $G$ as a permutation group on the unordered sets of $k-1$ points we obtain

$$
|G|=\left(\begin{array}{c}
n \\
k-1
\end{array}\right) b \quad \text { where } \quad b=\left|G_{(\Sigma)}^{\Sigma}\right| .
$$


Using the fact that $G$ is $(k-2)$-transitive we obtain

$$
|G|=\left(\begin{array}{c}
n \\
k-2
\end{array}\right)(k-2) ! h \quad \text { where } h=\left|G_{[k-2]}\right| .
$$

From Eqs. (1) and (2) we obtain

$$
b=\frac{n-k+1}{k} a
$$

and from Eqs. (2) and (3) we obtain

$$
\frac{n-k+2}{(k-1) !} b=h .
$$

We must now consider separately the different values of $k$.

(a) $k=3$. Clearly $b=1$ or 2 . If $b=2$, we may interchange any two points and $G$ is 2 -transitive. Hence $b=1$. Eq. (4) now gives $3=(n-2) a$, hence $a=1$ or 3. If $a=1$, then $n=5$ and from Eq. (5) it follows that $h=2$. Again, this implies that two points may be interchanged, which is impossible. Hence $a=3$ and $n=3$. Clearly, in this case $G=A^{2}$.

(b) $k=4$. Eq. (4) becomes

$$
b=\frac{n-3}{4} a
$$

and Eq. (5) becomes

$$
\frac{n-2}{6} b=h .
$$

Clearly $b=1,2,3$, or 6 . If $b=6$, then $G$ is 3 -transitive. We may now readily verify that the only integral solutions of Eqs. (6) and (7) for the other values of $b$ are:

$$
\begin{array}{llll}
b=2, & a=1, & n=11, & h=3, \\
b=2, & a=4, & n=5, & h=1, \\
b=3, & a=4, & n=6, & h=2, \\
b=3, & a=12, & n=4, & h=1 .
\end{array}
$$

In case (1), since $h=3$, some permutation will permute three points cyclically. Consequently $3 \mid b$, which is a contradiction.

In case (3), since $h=2$, some permutation fixes two points and transposes two other points. By taking $\Sigma$ to consist of one of these fixed points and the pair of transposed points we have that $2 \mid b$, which is a contradiction.

Cases (4) and (2) can occur and, as is easily verified, correspond respectively to $A^{\Omega}$ and the exceptional case (i) of the lemma.

(c) $k=5$. Eqs. (4) and (5) become respectively

$$
b=\frac{n-4}{5} a
$$


and

$$
\frac{n-3}{24} b=h
$$

We shall also need the following result due to GorEnsteIn and HugHes [7]:

If $G$ is a 3 but not 4-transitive group on a set $\Omega$ of $n$ points and $G$ is 4regular then either

(I) $G$ is sharply 3-transitive or

(II) $G=P \Gamma L_{2}\left(2^{q}\right)$ where $q$ is an odd prime and $n=2^{q}+1$.

We shall deal separately with these two possibilities.

(I) $G$ is sharply 3-transitive. Clearly $h=1$. We may now readily verify that the only integral solutions of Eqs. (8) and (9) are:

$$
\begin{array}{ll}
b=4, & n=9 . \\
b=6, & n=7 . \\
b=8, & n=6 . \\
b=12, & n=5 .
\end{array}
$$

Case (2) cannot occur, since the degree of a sharply 3-transitive group is one more than a power of a prime (see for example BURNSIDE [2], p. 178), and 7 is not of this form.

Case (1) can occur and corresponds to the exceptional case (iii) of the lemma. This is easily seen as follows. It is well known (see for example CoLE [3]) that there is a unique sharply 3-transitive group of degree 9, namely $P G L_{2}$ (8). It is easily verified that the subgroup fixing an unordered set of three points is transitive on the remaining six points. Hence the group is $4^{*}$-transitive and consequently also $5 *$-transitive.

Case (3) can occur and clearly corresponds to the exceptional case (ii) of the lemma.

Case (4) corresponds to $G=A^{\Omega}$.

(II) $G$ is $P \Gamma L_{2}\left(2^{q}\right)$. In this case $|G|=n(n-1)(n-2) q$. Eq. (3) shows that $q=h$. Eq. (9) now becomes

$$
\left(2^{q}-2\right) b=24 q \text {. }
$$

If $q=3$, then $n=9$. Since $P \Gamma L_{2}(8)$ contains $P G L_{2}(8)$ it must also be $4 *$ and $5^{*}$-transitive. This case corresponds to the exceptional case (iv) of the lemma.

If $q=5$, then $n=33$ and $b=4$. However these values contradict Eq. (8).

If $q=7$, then Eq. (10) has no solution for $b$.

If $q>7$, the left hand side of Eq. (10) is greater than the right hand side.

(d) $k=6$. We shall need the following result: the only 4 but not 5 -transitive and 5-regular groups are $A_{6}$ and $M_{11}$. 
This is an immediate consequence of the result of Gorenstein and Hughes mentioned above and the following extension due to $\mathrm{M}$. Hall of a theorem of Jordan (see for example HaLL [8] p. 73): the only 4-transitive groups where the stabilizer of 4 points has odd order are $S_{4}, S_{5}, A_{6}, A_{7}$ and $M_{11}$.

The case $n=11$ and $G=M_{11}$ cannot occur since using Eq. (2) we have

$$
|G|=11 \cdot 10 \cdot 9 \cdot 8=\frac{11 \cdot 10 \cdot 9 \cdot 8 \cdot 7}{5 !} b
$$

which leads to a contradiction. Hence $n=6$ and $G=A^{2}$ is the only possibility.

(e) $k=7$. It follows from the last section that a 5 but not 6-transitive and 6-regular group is either $A_{7}$ or a sharply 5-transitive group of degree 12 . As in the previous case the use of Eq. (2) eliminates the second possibility.

(f) $k \geqq 8 . M_{12}$ has no transitive extension, since no primitive group of degree 13 other than $A_{12}$ or $S_{12}$ has order divisible by 5 (see BURNSIDE [2] p. 213). Hence the only $(k-2)$ but not $(k-1)$-transitive and $(k-1)$-regular group, where $k \geqq 8$, is $A_{k}$.

This completes the proof of Lemma 9.

Since the conditions of Lemma 9 imply that $2 k>n$, Theorem 2 (a) follows from Theorem 1 and Lemma 8 by induction on $k$.

The result of Beaumont and Peterson referred to in the introduction is also an immediate consequence of our Lemmas 7 and 9.

\section{§ 5. Proof of Theorem 2 (b)}

Our proof will be based on showing that for $k \geqq 5$ the group $G$ acts on some set of $k$ letters as $S_{k}$.

Theorem 3. Let $k \geqq 1$, and let $G$ be a $k$-fold transitive group on a set $\Omega$ of $n$ points. If $G$ is not $(k+1)$-regular there exists a subset $\Pi$, with $n>|\Pi|>k$, such that $G_{(I)}$ is $k$-transitive on $\Pi$.

Proof. Let $\Delta$ be a subset of $k$ points of $\Omega$. Then since $G$ is not $(k+1)$ regular there exists a point $\alpha \in \Omega-\Delta$ such that $G_{[\Delta, \alpha]} \neq 1$. Let $p$ be a prime which divides $\left|G_{[\Delta, \alpha]}\right|$. Further, let $S$ be a Sylow $p$-subgroup of $G_{[\Delta]}$. There are now two cases to consider.

(I) $S$ fixes points not in $\Delta$. In this case we take $\Pi$ to be the set of all the points of $\Omega$ left fixed by $S$. By a theorem of MiLLER (see for example HaLL [8] p. 68) $G_{(\Pi)}$ is $k$-transitive on $\Pi$.

(II) $S$ fixes only the points of $\Delta$. Let $S$ split the points of $\Omega-\Delta$ into orbits of lengths $p^{a_{1}}, \ldots, p^{a_{s}}$, where $1 \leqq a_{1} \leqq \cdots \leqq a_{s}$. Also let $|S|=p^{b}$. Then, if $\beta$ is a point of an orbit of length $p^{a_{1}}$, it follows that $\left|S_{[\beta]}\right|=p^{b-a_{1}}$.

Now let $\Pi$ be the set of all points left fixed by $S_{[\beta]}$. From our choice of $p$ it follows that $|\Pi|<n$. Let $\Gamma$ be any subset of $k$ points of $\Pi$. Clearly $S_{[\beta]}$ is a subgroup of $G_{[\Gamma]}$ and since $S_{[\beta]}$ is a $p$-group but not a Sylow $p$-subgroup of 
$G_{[\Gamma]}$ there exists a $p$-group $C$ in $G_{[\Gamma]}$ which contains $S_{[\beta]}$ as a normal subgroup of index $p$. Since $S_{[\beta]}$ is a normal subgroup of $C$ and $\Pi$ is the fixed set of $S_{[\beta]}$ it follows that $C$ maps $\Pi$ onto itself.

We now wish to show that $C$ fixes only the points of $\Gamma$. Let us assume that $C$ fixes a point $\varepsilon$ not in $\Gamma$. Then if $T$ is a Sylow $p$-subgroup of $G_{[r]}$ containing $C$ let the point $\varepsilon$ lie in an orbit of $T$ of length $p^{e}$. Clearly $\left|T_{[\varepsilon]}\right|=p^{b-e}$ and since $C \subseteq T_{[\varepsilon]}$ it follows that $p^{b-a_{1}+1} \mid p^{b-e}$, i.e., $e<a_{1}$. Hence $T$ has a shorter orbit than $S$ which is impossible since $S$ and $T$ are conjugate in $G$. Thus $C$ is a permutation group of $\Pi$ fixing only the points of $\Gamma$. We may now apply Lemma 6 to complete the proof.

By repeated application of this theorem we obtain:

Corollary. There exists a subset $\Sigma$ of $\Omega$, with $n>|\Sigma|>k$, such that $G_{(\Sigma)}$ is $k$-transitive and $(k+1)$-regular on $\Sigma$.

Lemma 10. Let $G$ be a $k$-transitive group on a set $\Omega$ of $n$ points, with $n>k \geqq 4$. Then there exists a subset $\Pi$ of $k+1$ points such that $G_{(I)}^{I} \supseteq A^{\Pi}$.

Proof. If $G=S^{\Omega}$ the result is obvious. Hence we may now assume without loss of generality that $G$ is not $(k+1)$-transitive. We shall treat separately the various cases which may arise.

(I) $G$ is $(k+1)$-regular.

(a) $k=4$. As was noted in $\S 4$, the only groups of this kind are $A_{6}$ and $M_{11}$. It was shown by FrobenIUs [6] that $M_{11}$ acts as $S_{5}$ on some subset of 5 points (Alternatively we may use a simple counting argument on the unordered sets of 5 points to show that $M_{11}$ splits these into at most 7 orbits and acts on a set of the shortest orbit as $A_{5}$ or $S_{5}$. This suffices for our purpose).

(b) $k=5$. The only groups of this kind are $A_{7}$ and $M_{12}$ (see for example HaLl [8] p. 80). Again, it was shown by FrobenIus [6] that $M_{12}$ acts as $S_{6}$ on some set of 6 points. (The same argument indicated in case (a) will show that $M_{12}$ acts as $A_{6}$ or $S_{6}$ on some set of 6 points).

(c) $k \geqq 6$. In this case $G=A^{\Omega}$.

(II) $G$ is not $(k+1)$-regular. The proof in this case follows immediately from the corollary of Theorem 3 and case I above.

We may now finish the proof of Theorem 2(b). Since $G$ is, by assumption, $k^{*}$-transitive it is clearly sufficient to show that for $k \geqq 5$ there exists a set $\Pi$ of $k$ points such that $G_{(I)}^{I}=S^{I}$. Theorem 2(a) and Lemma 10 have shown that $G_{(I)}^{I I} \supseteq A^{I}$, hence it only remains to show that $G_{(I)}^{I I}$ contains an odd permutation.

We know that $G$ is 4-transitive and has degree at least 10. Let $\Delta$ be a subset of 3 points. Suppose first that $G_{[4]}$ has odd order. Then the stabilizer of 4 points has a fortiori also odd order, hence by M. HALL's theorem quoted in $\S 4$ (d) the group must be $M_{11}$. However, in $M_{11}$ the stabilizer of 3 points has even order, which is a contradiction. Hence $G_{[\Delta]}$ has even order. Let $a$ 
be an element of order 2 in $G_{[A]}$. We now select $\Pi$ to consist of an odd number of pairs of points interchanged by $a$ and of points fixed by $a$. Clearly $a$ restricted to $\Pi$ is an odd permutation.

This completes the proof of Theorem 2 .

\section{§ 6. Some examples of $\boldsymbol{k}^{*}$-transitive groups}

We append in this paragraph some remarks on groups which are $k^{*}$ transitive but not $k$-transitive. We shall say that a group is of type $k^{*}$ if it is $k^{*}$ but not $k$-transitive and has degree at least $2 k$. Then Theorem 2 (b) shows that groups of type $k^{*}$ can exist at most for $k=2,3$ and 4 . We discuss these cases separately and give some examples for each case.

(a) $k=2$. A group of type $2 *$ contains no permutation which interchanges two points. It is therefore of odd order and hence soluble (FeIT and THOMPSON [4]). Also every $2 *$-transitive group is primitive, since if $\alpha$ and $\beta$ were two points in one block and $\gamma$ a point in another the group would contain a permutation carrying the pair $\alpha, \beta$ to the pair $\alpha, \gamma$. Hence we may write $n=p^{v}, p$ an odd prime, and we know that the group is contained in $A_{v}(p)$. Also, since the group is transitive on the $\left(\begin{array}{l}n \\ 2\end{array}\right)$ unordered pairs, $p \equiv-1(4)$ and $v$ is odd.

The groups of type $2^{*}$ can be characterized as those transitive groups of degree greater than three and of odd order in which the subgroup fixing one point has exactly two orbits on the rest. That the condition is necessary follows from the above remarks and the observation that the group has exactly two orbits on the ordered pairs, since if $\alpha, \beta$ are any two points any pair can be mapped to $(\alpha, \beta)$ or to $(\beta, \alpha)$. It is clearly sufficient.

Using this condition it is immediate that if $q \equiv-1$ (4) then the group of all permutations of the elements of the field $F_{q}$ given by $x \rightarrow a x+b$ with $a$ a square is of type $2^{*}$.

(b) $k=3$. It is easy to determine the soluble groups of type $3^{*}$. The doubly transitive soluble groups have been described by Huppert [10]. Apart from some exceptional groups of known order and degree they are all transitive subgroups of the groups $\Gamma A_{1}(q)$. Comparison of the orders and degrees shows that the exceptional groups are not $3 *$-transitive. It will now suffice to determine the values of $q$ for which $\Gamma A_{1}(q)$ is $3 *$-transitive.

$\Gamma A_{1}(q), q=p^{\nu}$, is 2-transitive and the subgroup fixing the two elements 0,1 of $F_{q}$ is the group of automorphisms of $F_{q}$. Hence this group has orbits of equal length on the remaining symbols if and only if $v=1$, or $p=2$ and $v$ is a prime.

If $v=1$ and $\Gamma A_{1}(q)$ is $3 *$-transitive then

$$
\left(\begin{array}{l}
p \\
3
\end{array}\right) \mid p(p-1)
$$

and $p \leqq 6$ and prime. These conditions are incompatible. 
If $p=2$ the condition

$$
\left(\begin{array}{l}
q \\
3
\end{array}\right) \mid v q(q-1)
$$

shows that $2^{v}-2 \mid 6 v$. Hence $v=3$ or 5 . Examination of the individual groups shows that $\Gamma A_{1}(32)$ is transitive and regular on the sets of three points and that $\Gamma A_{1}(8)$ is also of type $3^{*}$. Thus it is only in the latter case that it is necessary to consider subgroups. $A_{1}(8)$ is transitive and regular on the sets of three points.

An example of an infinite family of non-soluble groups of type $3 *$ is provided by the groups $P S L_{2}(q), q>3, q \equiv-1(4)$.

(c) $k=4$. By reasoning similar to the above it may be verified that, among the groups $P \Gamma L_{m}(q)$ and their transitive subgroups, there occur precisely three groups of type $4^{*}$, viz. $P \Gamma L_{2}(8), P \Gamma L_{2}(32)$ and $P G L_{2}(8)$.

\section{References}

[1] Beaumont, R.A., and R.P. Peterson: Set-transitive permutation groups. Can. J. Math. 7, 35- 42 (1955).

[2] BuRnsIDE, W.: Theory of groups of finite order. Cambridge: University Press 1911.

[3] Cole, F.N.: List of the substitution groups of nine letters. New York M.S. Bull. 2, $250-258$ (1893).

[4] Feit, W., and J.G. Thompson: Solvability of groups of odd order. Pacific J. Math. 13, $775-1029$ (1963).

[5] Frobentus, G.: Über die Charaktere der symmetrischen Gruppe. Sitzungsber. Akad. Berlin 1900, S. 516-534.

[6] - Uber die Charaktere der mehrfach transitiven Gruppen. Sitzungsber. Akad. Berlin 1904 , S. 558- 571 .

[7] GoRensteIn, D., and D.R. Hughes: Triply transitive groups in which only the identity fixes four letters. Ill. J. Math. 5, 486-491 (1961).

[8] HaLl jr., Marshall: The theory of groups. New York: MacMillan 1959.

[9] Hughes, D.R.: On $k$-homogeneous groups. Arch. Math. 15, 401- 403 (1964).

[10] HuPpert, B.: Zweifach transitive, auflösbare Permutationsgruppen. Math. Z. 68, $126-150$ (1957).

[1I] JACOBI, C.G.J.: De functionibus alternantibus earumque divisione per productum e differentiis elementorum conflatum. Crelle Journal 22, 360-371 (1841).

[12] Mrller, G.A.: Multiply transitive groups. Trans. Amer. Math. Soc. 28, 339-345 (1926).

[13] WIELANDT, H.: Finite permutation groups. New York and London: Academic Press 1964.

[14] - Unendliche Permutationsgruppen. (Lecture notes.) Math. Inst. Univ. Tübingen 1960.

Department of Mathematics, University of Michigan, Ann Arbor, Mich. (U.S.A.)

Queen Elizabeth College, London (England)

(Received December 21, 1964) 